\title{
GENERALIZED EIGENFUNCTION EXPANSIONS FOR OPERATOR ALGEBRAS $\left({ }^{(1)}\right.$
}

\author{
BY \\ R. T. HARRIS
}

A generalized eigenfunction expansion may be regarded as a representation for the spectral theorem by a transform technique. These representations have been presented in many forms, an early version of which was the von Neumann "direct integral" decomposition for a class of operator algebras [19]. In 1953 [17], Mautner applied the von Neumann technique to the class of operators acting in an $L^{2}$-space whose spectral projections corresponding to the bounded Borel sets are represented by Carleman kernels. These results were also obtained by Bade and Schwartz [1] in 1956 through the use of the Dunford-Pettis theorem [8]. Also, Nelson [18] in 1958 obtained these results in the form of an operator decomposition by a kernel representation of the operators involved. Using the techniques of Mautner, Browder and Gårding $[3 ; 9]$ in 1954 were the first to obtain an eigenfunction expansion for any self-adjoint realization of an elliptic partial differential operator. A somewhat different technique was introduced in 1955 when Gelfand and Kostyučenko obtained a general theorem for the existence of an eigenfunction expansion for a self-adjoint realization of any partial differential operator. Their proof employed a vector-valued differentiation theorem, proved first for Hilbert spaces by Birkhoff [2] and for general reflexive Banach spaces by Gelfand [11]. In 1956, Gelfand and Silov [13] seemed to have been the first to observe the vast generality of these existence theorems and the possibility of a semi-algebraic formulation of the basic hypotheses used. Most striking, perhaps, was the fact that the existence theorem for an eigenfunction expansion could be completely independent of the type of operator considered, and a function only of the topological nature of the subspace over which the expansion takes place. Gelfand and Silov essentially use the nuclearity of the space over which the expansion exists to verify the hypotheses of the GelfandBirkhoff differentiation theorem.

All of the existence theorems for generalized eigenfunction expansions mentioned above have been highly analytical. A focal point of our investigation might be regarded as the attempt to replace these analytical techniques by the semi-algebraic techniques suggested by Grothendieck's thesis [14] and Gelfand and Šilov [13]. Our results, in essence, prove that in the context

Presented to the Society, November 21, 1959 under the title Eigenfunction expansions for operator algebras; received by the editors September 15, 1959 and, in revised form, July 5, 1960.

(1) This paper is essentially the author's dissertation, University of Illinois, October 1959, work on which was supported by National Science Foundation Contract G-7011. 
of the spectral theorem, the hypotheses for the applicability of the GelfandBirkhoff theorem are equivalent to certain maps being "nuclear"-a condition that is ofttimes much easier to verify. For generalized eigenfunction expansions, we are able to remove the hypothesis of reflexivity from the Gelfand-Birkhoff theorem as well as generalize the known existence theorems to arbitrary abelian uniformly closed ${ }^{*}$-algebras of operators with the identity in the weak closure. In this situation, the Gelfand-Birkhoff theorem can be obtained as a consequence of the Dunford-Pettis theorem [8].

In Part I, we give basic definitions concerned with the general spectral theorem and prove the major measure-theoretic result that we require. In Part II, the definition of a "generalized eigenfunction expansion" is given, and its elementary properties are estabished. In Part III, we establish the equivalence of various hypotheses for the existence of generalized eigenfunction expansions. Part IV introduces a generalized operator decompositionanalogous to the Weyl "matrix measure" for an ordinary differential operator -and establishes conditions for its existence. We conclude with a short proof of a theorem of Gelfand and Silov [13].

\section{Preliminaries}

1. The Gelfand isomorphism. Let $H$ denote some fixed separable Hilbert space. The algebra of bounded linear operators acting on $H$ will be denoted by $L(H)$. We let $V$ always denote some locally compact space denumerable at infinity and $C_{0}(V)$, the space of complex-valued continuous functions vanishing at infinity over $V$. We give $C_{0}(V)$ the structure of a Banach space through the norm given by the supremum of a function in $C_{0}(V)$; with this norm, $C_{0}(V)$ is a $B^{*}$-algebra with complex conjugation as the adjoint. It is well known [7, Appendix I] that a uniformly closed abelian *-algebra (which is a $B^{*}$-algebra, cf. [7, p. 2]) $A$ of operators acting on $H$ may be identified with the range of a ${ }^{*}$-isomorphism taking $C_{0}(V)$ into $L(H)$ where $V$ may be identified with the set of regular maximal ideals of the algebra $A ; V$ is called the spectrum of $A$. The denumerability of $V$ at infinity is a consequence of the separability of $H$. The map carrying $C_{0}(V)$ onto $A$ we call the Gelfand Isomorphism, which we denote by $G$. For notational convenience, we write $T_{f}$ for $G(f)$. We always assume that the identity operator is in the weak closure of $A$.

For $x$ and $x^{\prime}$ two given elements of $H$, we define a map $m_{x, x^{\prime}}$ from $C_{0}(V)$ to the complex numbers by sending an element $f$ of $C_{0}(V)$ into $\left\langle T_{f} x, x^{\prime}\right\rangle$, the brackets denoting the inner product in $H$. It is easily seen that $m_{x, x^{\prime}}$ is a complex Radon measure over $V$.

For some fixed vector $x$ in $H$, we define a map from $C_{0}(V)$ into $H$ by assigning to $f$ the element $T_{f} x$. If $C_{0}(V)$ is given the Hermitian form $[f \mid g]=\int_{V} f \bar{g} d m_{x, x}$, then the completion of $C_{0}(V)$ with the topology introduced by this form is $L^{2}\left(m_{x, x}\right)$. Since $\left\langle T_{f} x, T_{g} x\right\rangle=\left\langle T_{f_{\bar{g}}} x, x\right\rangle=\int_{V} f \bar{g} d m_{x, x}$, we infer that the map $f$ to 
$T_{f} x$ is an isometry; we denote its extension to $L^{2}\left(m_{x, x}\right)$ by $\mathbb{C}^{*}$. The adjoint of $\mathfrak{S}^{*}$, which we denote by $\mathfrak{S}$, sends $H$ into $L^{2}\left(m_{x, x}\right)$, and $m_{y, x}=\mathfrak{S}(y) \cdot m_{x, x}$.

For the convenience of horizontal notation, we will suppress the subscript " $x$ " wherever possible and call $\{x, \mathfrak{S}, m\}$ a cyclic system, taking it for granted that 5 and $m$ depend upon an implicit element $x$ in $H$.

We also recall that there is an extension of the Gelfand isomorphism to the bounded Borel functions over $V$ (cf. [7, Appendix $I ; 18$, p. 93]). If $B$ is a Borel set of $V$, then $E_{B}$ will denote the image of the characteristic function of $B$.

2. A theorem on the existence of an upper bound for a family of measures. We now determine conditions for certain subfamilies of $\left\{m_{x, x^{\prime}} \mid x, x^{\prime}\right.$ in $\left.H\right\}$ to have an upper bound. This procedure replaces the customarily-used GelfandBirkhoff differentiation theorem $[11 ; 2]$ in the proof of the existence of an eigenfunction expansion from a bounded variation condition on the spectral measure (cf., for example, [4]).

THEOREM 1. Let $V$ be a locally compact space and let $M_{b}(V)$ be the space of bounded real measures over $V$. Let $F$ be a family of positive measures in $M_{b}(V)$ and assume that there exists a map from the Borel sets of $V$ to the reals having the following properties:

(a) If $A$ and $B$ are Borel sets of $V$, then $X(A \cup B) \leqq X(A)+X(B)$.

(b) If $A$ and $B$ are Borel sets of $V$ with $A$ contained in $B$, then $X(A) \leqq X(B)$. Also, for the void set, $\varnothing$, we have $X(\varnothing)$ equals 0 .

(c) For a given Borel set $A$ of $V$ and a given $\epsilon>0$, there exists an open set $U$ containing $A$ such that $X(U) \leqq X(A)+\epsilon$.

(d) For any Borel set $A$ of $V$ and any measure $m$ in $F$, we have $X(A) \geqq m(A)$.

(e) There exists some constant $k$ such that for any finite collection, $\left\{A_{i}\right\}$, of disjoint Borel sets of $V$, we have $\sum_{i} X\left(A_{i}\right) \leqq k$.

Then, the family $F$ is bourded in the lattice structure of $M_{b}(V)$.

Proof. It is sufficient to show that there exists a positive measure $v$ such that for any compact set $K, v(K) \geqq X(K)$. Assume that some uniform structure is chosen compatible with the topology of $V$. We denote a given element in the uniformity by $\theta$. The set of finite coverings of the compact set $K$ by open sets each of which is small of order $\theta$ will be denoted by $C(K, \theta)$. Let $c$ be an element of $C(K, \theta)$ given by the finite indexed family of open sets $\left\{U_{i}\right\}$. Set $T(c)=\sum X\left(U_{i}\right) ; T$ is a mapping from $C(K, \theta)$ to the reals. We note the implicit dependence of $T$ upon $K$. For each $\theta$, the infimum of all numbers $T(c)$ for $c$ in $C(K, \theta)$ will be denoted by $\mathfrak{h}(K, \theta)$ (the "mass" of $K$ using coverings small of order $\theta$ ). Now for each fixed $K$, we take the supremum of the family $\mathfrak{h}(K, \theta)$ over all elements $\theta$ in the given uniformity, and we call this number (finite or infinite) $\mathfrak{h}(K)$. We obtain a set function, which we denote by $\mathfrak{h}$, defined over the compact sets of $V$. We show that $\mathfrak{h}$ satisfies the following properties: 
For arbitrary $K$, (i) $\mathfrak{h}(K)$ is finite, (ii) $\mathfrak{h}(K) \geqq X(K)$, and (iii) the set function $\mathfrak{h}$ extends to a measure $v$.

(i) For a given $a>0$, we show that for any $\epsilon$, there exists a $c$ in $C(K, \theta)$ such that $T(c)<\operatorname{Var} X+a$; for, we may conclude from this that $\mathfrak{h}(K)$ $\leqq \operatorname{Var} X$, and this last is finite by (e). For an arbitrary $\theta$, there exists a partition of $K$ with Borel sets small of order $\theta$. Let $\left\{A_{i}\right\}, 0 \leqq i \leqq N$, be such a family for $K$. Condition (c) states that there exists an open set $U_{i}$ containing $A_{i}$, small of order $\theta$, such that $X\left(U_{i}\right) \leqq X\left(A_{i}\right)+a / N$. This yields

$$
\sum_{i} X\left(U_{i}\right) \leqq \sum_{i} X\left(A_{i}\right)+a \leqq \operatorname{Var} X+a,
$$

that is to say, there exists a $c$ such that $T(c) \leqq \operatorname{Var} X+a$.

(ii) From sub-additivity and monotonicity of $X$, we have, for arbitrary $c$ in $C(K, \theta), T(c) \geqq X(K)$; that is, $\mathfrak{h}(K) \geqq X(K)$.

(iii) We now show there exists a bounded measure $v$ such that for any compact set $K$ of $V, v(K)=\mathfrak{h}(K)$.

Boundedness follows from the fact that $v$ is an extension of $\mathfrak{h}$, since for all compact $K$ of $V, \mathfrak{h}(K) \leqq \operatorname{Var} X$. That $\mathfrak{h}$ is monotone is clear, since if $K$ is contained in $K^{\prime}$, then $C(K, \theta)$ contains $C\left(K^{\prime}, \theta\right)$. Sub-additivity of $\mathfrak{h}$ is also immediate.

To prove additivity over disjoint $K$ and $K^{\prime}$, we first note that there exists a $\theta_{0}$ such that every covering of $K \cup K^{\prime}$ small of order $\theta_{0}$ has the property that the union of the sets covering $K$ is disjoint from the union of the sets covering $K^{\prime}$. Whence, for $\theta$ finer than $\theta_{0}$, it follows that $\mathfrak{h}\left(K \cup K^{\prime}, \theta\right)$ is equal to the infimum of $T(c)+T\left(c^{\prime}\right), c$ and $c^{\prime}$ in $C(K, \theta)$ respectively; so, $\mathfrak{h}\left(K \cup K^{\prime}, \theta\right)$ $=\mathfrak{h}(K, \theta)+\mathfrak{h}\left(K^{\prime}, \theta\right)$. Since $\mathfrak{h}(K, \theta)$ is a monotone function of $\theta$ with the uniformity given its natural filtration by inclusion, the suprema may be replaced by limits so that on using additivity of the limits, we have $\mathfrak{h}\left(K \cup K^{\prime}\right)=\mathfrak{h}(K)$ $+\mathfrak{h}\left(K^{\prime}\right)$.

Finally, we show that for a given compact set $K$ of $V$ and a given $a>0$, there exists an open set $U$ containing $K$ such that for all $K^{\prime}$ contained in $U$, $\mathfrak{h}\left(K^{\prime}\right) \leqq \mathfrak{h}(K)+a$.

First, for every $a>0$ there exists an open set $U$ containing $K$ such that for all $K^{\prime}$ contained in $(V-K) \cap U$, we have $\mathfrak{h}\left(K^{\prime}\right)<a$. Assume the contrary. Then, there exists a sequence of compact sets $K_{n}$ such that $\mathfrak{h}\left(K_{n}\right) \geqq a$, and a decreasing sequence of open pre-compact sets $U_{n}$ such that $U_{n}$ contains $K$, $K_{n}$ is contained in $(V-K) \cap U_{n}$, and $K_{n-1}$ is contained in the complement of the closure of $U_{n}$. The existence of such a sequence follows from an inductive argument by first letting $U_{1}$ be some open pre-compact set containing $K$ and letting $K_{1}$ be some compact set in $(V-K) \cap U_{1}$ with the property that $\mathfrak{h}\left(K_{1}\right) \geqq a$. Assume that the sequence is defined for all $m \leqq n-1$. Using the fact that $K_{n-1}$ does not intersect $K$, there exists an open set $U_{n}$ containing $K$ and contained in $U_{n-1}$ whose closure does not intersect $K_{n-1}$. By the hypothesis of contradiction, there exists a $K_{n}$ in $U_{n}$ not intersecting $K$ such that 
$\mathfrak{h}\left(K_{n}\right) \geqq a$. This completes the construction. We remark that the sequence of compact sets $K_{n}$ forms a mutually disjoint family since the sets $U_{n}$ are decreasing. Now, we define another sequence of compact sets $H_{n}=\bigcup_{0<i \leq n} K_{i}$; since $\mathfrak{h}$ is additive over disjoint sets, $\mathfrak{h}\left(H_{n}\right) \geqq n a$. This is impossible since $\mathfrak{h}\left(H_{n}\right) \leqq \mathfrak{h}\left(\bar{U}_{1}\right)$ by monotonicity and the fact that $\bar{U}_{1}$ is compact so that $\mathfrak{h}\left(\bar{U}_{1}\right)$ is finite.

Now, let $U$ be such an open set containing $K$ having the property that for any compact set $H$ contained in $U \cap(V-K), \mathfrak{h}(H) \leqq a / 3$. There exists some $c$ in $C(K, \theta)$ such that $T(c) \leqq \mathfrak{h}(K, \theta)+a / 3$. Letting $M$ be the union of all the open sets in $c$ and setting $H$ equal to $K^{\prime} \cap(V-M), K^{\prime} \subseteq U$, there exists a $c^{\prime}$ in $C(I, \theta)$ such that $T\left(c^{\prime}\right) \leqq \mathfrak{h}(H, \theta)+a / 3$. The union of the elements of $c$ and $c^{\prime}$ covers $K^{\prime}$ - so that $T(c)+T\left(c^{\prime}\right) \leqq \mathfrak{h}(K \cup H, \theta)+(2 / 3) a \leqq \mathfrak{h}(K, \theta)+a$ by the defining property of $U$. Hence, we have $\mathfrak{h}\left(K^{\prime}, \theta\right) \leqq \mathfrak{h}(K, \theta)+a$ so that $\mathfrak{h}\left(K^{\prime}\right) \leqq \mathfrak{h}(K)+a$, which was to be proved.

By $[6$, Chapter 3, p. 165 , Theorem $5 ; 15, \S \S 53,54$, in particular Theorem $A, \S 54], \mathfrak{h}$ extends to a positive measure which we call $v$. We have already remarked that $v$ must be bounded, so $v$ is an element of $M_{b}(V)$ and $v$ is a bound for the family $F$.

\section{The FUndamental DEFinitions AND THEIR GENERAL PROPERTIES}

In what follows, all linear topological spaces will be assumed to be locally convex and separated (i.e., Hausdorff). If $E$ is such a space, then $E^{\prime}$ will denote the antidual, that is, the space of conjugate linear functionals over $E$, given the strong topology-the topology of uniform convergence over the bounded sets of $E$.

\section{Refining subspaces of $H$.}

Definition 1. We will call a linear topological space $D$ a refining space for $H$, if the following conditions are satisfied:

(a) As a vector space $D$ is contained in $H$.

(b) The map $i: D \rightarrow H$ induced by inclusion is continuous and has dense range in $H$.

(c) The space $D^{\prime}$ is separable.

It is clear that if $D$ is reflexive and satisfies conditions (a) and (b) of Definition 1 , then $D$ is a refining space for $H$.

We regard $H^{\prime}$ as a subspace of $D^{\prime}$ through the imbedding given by the adjoint of $i, i^{*}: H^{\prime} \rightarrow D^{\prime}$. For convenience of notation, we omit the " $i^{*}$ " where it is possible to do so without confusion.

2. The articulation of a cyclic system over a refining space. Let $K$ be a mapping from $V$ to the antidual $E^{\prime}$ of some linear topological space $E$. For any $\lambda$ in $V, K_{\lambda}$ will denote the value of $K$ at $\lambda$. Let $m$ be a positive measure over $V$. If for each $e$ in $E$, the function sending $\lambda$ to $\left\langle e, K_{\lambda}\right\rangle$ is measurable, then we say $K$ is $w^{*}$-measurable. We denote the a.e. equivalence class of functions formed from the map taking $\lambda$ to $\left\langle e, K_{\lambda}\right\rangle$ by $\langle e, K\rangle$. 
Definition 2. A cyclic system $\{x, \mathfrak{E}, m\}$ determined by the operator algebra $A$ will be called articulate over a refining space $D$ for $H$ if $D$ is a Banach space and if there exists a map $K$ taking the spectrum $V$ of $A$ into $D^{\prime}$ having the following properties:

(a) The scalar function $\lambda \rightarrow\left|K_{\lambda}\right|$ is $m$-integrable.

(b) For each $a$ in $D$, $\mathfrak{S}(a)=\langle a, K\rangle$.

Definition 2 is the precise sense we attach to the phrase "generalized eigenfunction expansion for $A$."

We note (b) implies that $K$ is $w^{*}$-measurable since $\mathbb{(}(a)$ is in $L^{2}(m)$.

We recall that a map taking $V$ into some linear topological space $E$ is measurable with respect to some positive measure $m$ over $V$ if, for a given $\epsilon>0$ and a compact set $H$ of $V$, there exists a compact set $H_{1}$ contained in $H$ such that the measure of the complement of $H_{1}$ in $H$ is less than $\epsilon$ and the restriction of the map to $H_{1}$ is continuous.

Proposition 1. If $\mathbb{C}$ is articulate over a refining space $D$, then the map $K$ sending $V$ into $D^{\prime}$ is measurable.

Proof. This a consequence of the following lemma due to Pettis [14, Chapter I, p. 21].

Lemma. Let $D$ be a Banach space with a separable antidual $D^{\prime}$ and let $K$ be a $w^{*}$-measurable map from $V$ to $D^{\prime}$. Then, $K$ is measurable.

Proposition 2. Let us assume that $D$ refines $H$ and that $(5$ is articulate over $D$. Then, for any $f$ in $L^{2}(m)$,

$$
\mathfrak{S}^{*}(f)=\int_{V} f k d m
$$

where the integral is the weak integral with respect to the pairing between $D$ and $D^{\prime}$.

Proof. Let $a$ be an arbitrary element of $D$. We have

$$
\left\langle a, \mathfrak{\complement}^{*}(f)\right\rangle=\int_{V} \mathfrak{E}(a) f d m=\int_{V}\langle a, K\rangle f d m=\left\langle a, \int_{V} K f d m\right\rangle,
$$

the last equality following from the definition of the weak* integral. Hence, we may write $\mathfrak{5}^{*}(f)=\int_{V} f K d m$.

Identifying both $D$ and $H$ with subspaces of $D^{\prime}$ through the maps $i$ and $i^{*}$ (cf. II, 1), let us assume that $\mathfrak{C}^{*}(f)$ is some element in $D$ which we relabel $a$. Then, $f=\langle a, K\rangle$, so that the weak* integral representation in Proposition 2 reads

$$
a=\int_{V} K\langle a, K\rangle d m
$$


PRoposicIon 3 (UNIQUENESS). Let (S be articulate over a refining space $D$ for $H$. Let $K$ and $K^{\prime}$ be two maps from $V$ to $D^{\prime}$ representing (5 as in Definition 2. Then, except for $\lambda$ in a set of m-measure zero, $K_{\lambda}=K_{\lambda}^{\prime}$.

Proof. By hypothesis, for all $a$ in $D,\left\langle a, K-K^{\prime}\right\rangle=0$. Since $D^{\prime}$ is separable, $D$ itself must be separable. Let $Q$ be countably dense in the sphere of $D$. For each $a$ in $Q$, let $W_{a}$ be the set of measure zero where $\left\langle a, K_{\lambda}-K_{\lambda}^{\prime}\right\rangle \neq 0$. Form the union of the sets $W_{a}$ for $a$ in $Q$ and call this set $W$. The fact that $Q$ is countable means that $W$ is a set of measure zero. Now, for any $\lambda$ in the complement of $W,\left|K_{\lambda}-K_{\lambda}^{\prime}\right|_{D^{\prime}}=\sup \left\{\left|\left\langle a, K_{\lambda}-K_{\lambda}^{\prime}\right\rangle\right| \mid a\right.$ in $\left.Q\right\}=0$. That is to say, except for $\lambda$ in a set of measure zero, $K_{\lambda}=K_{\lambda}^{\prime}$.

Denote by $H(x)$ the smallest closed subspace of $H$ which contains $x$ and is invariant under the action of $A$. Now $H$ may be decomposed into a countable number of such cyclic subspaces, that is, there exists a countable collection $\left\{x_{i}\right\}$ such that $\oplus_{i} H\left(x_{i}\right)=H$. The spectral theorem may be given in the form of a countable collection of cyclic systems $\left\{x_{i}, \mathfrak{S}_{i}, m_{i}\right\}$ each $\mathfrak{S}_{i}$ taking $H\left(x_{i}\right)$ isometrically onto $L^{2}\left(m_{i}\right)$. For such a collection of generators, we may form $\left\{x_{i}, \mathbb{C}_{i}, m_{i}\right\}$. If $D$ is a refining space over which each of the $\mathfrak{C}_{i}$ is articulate, then there exists a family of kernels $K_{i}$ mapping $V$ to $D^{\prime}$ such that the map $\mathfrak{C}_{i}$ from $D$ to $L^{2}\left(m_{i}\right)$ is given by $\mathfrak{S}_{i}(a)=\left\langle a, K_{i}\right\rangle$. Forming $\oplus_{i} L^{2}\left(m_{i}\right)$, we observe that the map sending $a$ in $D$ to $\sum_{i} \mathfrak{S}_{i}(a)$ in $\oplus_{i} L^{2}\left(m_{i}\right)$ extends to an isomorphism of $H$ onto $\oplus_{i} L^{2}\left(m_{i}\right)$.

\section{Conditions for the ARTiculation of A CYClic system over A GIVEN REFINING SPACE $D$}

We now show the equivalence of a set of conditions that have been used in the literature for the articulation of $\{x, \mathfrak{C}, m\}$ over some given refining space $D$. Essentially, we prove that the approach through the GelfandBirkhoff differentiation theorem is equivalent to certain semi-algebraic properties of (5).

If $E$ and $F$ are linear topological spaces, we denote by $L(E, F)$ the space of linear continuous maps from $E$ to $F$ given the topology of uniform convergence over the bounded sets of $E$.

Definition 2. Let $E$ and $F$ be Banach spaces and let $E \otimes F$ denote the tensor product of $E$ and $F$. If $y$ is an element of $E \otimes F$, then the number $|y|_{1}$ denotes the infimum of all $\sum_{i}\left|e_{i}\right|_{E}\left|f_{i}\right|_{F}$ where $y=\sum_{i} e_{i} \otimes f_{i}$. The realvalued function sending $y$ into $|y|_{1}$ is a norm over $E \otimes F$, and $E \otimes \wedge F$ will denote the completion of $E \otimes F$ in the topology induced by this norm. A nuclear map of $E$ into $F$ will be an element of $L(E, F)$ in the canonical image of $E^{\prime} \otimes{ }^{\wedge} F$ in $L(E, F)$.

We also recall that a family of functions defined over a space $V$ with a positive measure is called equimeasurable if for any compact set $H$ and $\epsilon>0$, there exists a compact set $K_{1}$ contained in $I I$ such that the measure of $H-K_{1}$ 
is less than $\epsilon$ and the restriction of the family to $K_{1}$ is equicontinuous (more precisely, each member of the family has a representative such that the family of representatives is equicontinuous).

We now state a theorem which gives explicit criteria for 5 to be articulate over a refining Banach space $D$.

TheOREM 2. Let $\{x, \mathfrak{S}, m\}$ be a cyclic system and $D$ a refining space for $H$. Then, the following are equivalent:

(a) 5 is articulate over $D$.

(b) (5 as a map from $D$ to $L^{1}(m)$ is nuclear.

(c) 5 takes bounded sets of $D$ into a family of $m$-integrable functions which is equimeasurable and bounded above in absolute value by an m-integrable function.

(d) (5 takes bounded sets of $D$ into a family of m-integrable functions which is bounded above in absolute value by an $m$-integrable function.

(e) There exists a constant $c$ such that for an arbitrary disjoint finite collection of Borel sets $\left\{B_{i}\right\}$ of $V$, then

$$
\sum_{i}\left|E_{B_{i}} x\right|_{D^{\prime}} \leqq c
$$

Proof. We note first that the articulating kernel $K$ is measurable (Proposition 1). Therefore, the equivalence of (a) through (c) is essentially a theorem of Grothendieck (cf. [14, Chapter 1, p. 64]).

That (c) implies (d) is obvious. We shall show that (d) implies (e). Let $\left\{B_{i}\right\}$ be an arbitrary disjoint finite collection of Borel sets of $V$. If $S_{D}$ denotes the unit ball in $D$, we have

$$
\begin{aligned}
\left|E_{B_{i}} x\right|_{D^{\prime}} & =\sup \left|\left\langle a, E_{B_{i}} x\right\rangle\right|=\sup \left|\int_{B_{i}} d m_{x, a}\right| \\
& =\sup \left|\int_{B_{i}} \subseteq(a) d m\right| \leqq \sup \int_{B_{i}}|\mathfrak{S}(a)| d m,
\end{aligned}
$$

where the above suprema are taken over all $a$ in $S_{D}$. Now, by hypothesis, there exists an $h$ in $L^{1}(m)$ such that $|\mathfrak{C}(a)| \leqq h$ for all $a$ in $S_{D}$. Therefore,

$$
\sup _{a \in S_{D}} \int_{B_{i}}|\mathfrak{S}(a)| d m \leqq \int_{B_{i}} h d m,
$$

that is,

$$
\left|E_{B_{i}} x\right|_{D^{\prime}} \leqq \int_{B_{i}} h d m
$$

On summing, we have, 


$$
\sum_{i}\left|E_{B_{i}} x\right|_{D^{\prime}} \leqq \sum_{i} \int_{B_{i}} h d m \leqq \int_{V} h d m<\infty .
$$

We now prove that (e) implies (a).

Let $F$ denote the family of measures $\left|\operatorname{Re} m_{a, x}\right|$ and $\left|\operatorname{Im} m_{a, x}\right|$ for $a$ in the unit ball of $D$. Define $X(A)$ to be the supremum of $\left|E_{B} x\right|_{D^{\prime}}$ for all $B$ contained in $A$. This determines a set function $X$ mapping the Borel sets of $V$ into the reals. With the hypothesis of (e), we now prove $X$ satisfies the hypotheses of Theorem 1 .

The verification of (a) and (b) is routine.

The assertion (e) follows from the hypothesis (e) above; for, if $\left\{A_{i}\right\}$ is an arbitrary finite family of disjoint Borel sets of $V$, we have

$$
\sum_{i} X\left(A_{i}\right)=\sum_{i} \sup _{B_{i} \subseteq A_{i}}\left|E_{B_{i}} x\right|_{D^{\prime}}=\sup _{B_{i} \subseteq A_{i}} \sum_{i}\left|E_{B_{i}} x\right|_{D^{\prime}} \leqq c .
$$

Since the map taking $D$ into $H$ is continuous, we have $|a|_{D} \leqq k|a|_{H}$ for some constant $k$ and arbitrary $a$ in $D$. By the Schwarz inequality,

$$
\begin{aligned}
\left|E_{B} x\right|_{D^{\prime}} & =\sup _{a \in S_{D}}\left|\left\langle a, E_{B} x\right\rangle\right| \leqq k\left|E_{B} x\right|_{H} \\
& \leqq k\left\langle E_{B} x, E_{B} x\right\rangle^{1 / 2} \leqq k(m(B))^{1 / 2}
\end{aligned}
$$

Taking the supremum on both sides for all $B$ contained in some $A$, we have $X(A) \leqq k(m(A))^{1 / 2}$. We infer from the continuity of the square root function and the regularity of $m$ that for given $\epsilon>0$ and a given Borel set $B$ of $V$, there exists an open set $U$ containing $B$ such that $(m(U-B))^{1 / 2} \leqq \epsilon / k$. As

$$
|X(U)-X(B)| \leqq X(U-B) \leqq k(M(U-B))^{1 / 2}
$$

we conclude that $|X(U)-X(B)| \leqq \epsilon$, or $X(U) \leqq X(B)+\epsilon$. This proves (c).

We are left with the proof of (d). Without loss in generality, we consider only the real parts. Since $X(B) \geqq\left|E_{B} x\right| D_{D^{\prime}} \geqq\left|\left\langle a, E_{B} x\right\rangle\right| \geqq\left|m_{a, x}(B)\right|$, on taking the suprema of both sides of the inequality $X(B) \geqq\left|m_{a, x}(B)\right|$ for all $B$ contained in $A$, we have that $X(A) \geqq\left|\operatorname{Re} m_{a, x}\right|(A)$. This completes the verification of the hypotheses of Theorem 1 .

It follows by Theorem 1 that the family $F$ of measures is bounded in the lattice structure of $M_{b}(V)$, the space of bounded measures over $V$. By the Schwarz inequality, we have $\left|m_{a, x}(A)\right| \leqq k(m(A))^{1 / 2}$. We infer from this that each of the measures in $F$ is absolutely continuous with respect to $m$. Equivalently [6, Chapter 5, Theorem 2, p. 47], the family $F$ is contained in the band generated by $m$, which we denote by $B(m)$. By the definition of $B(m)$ [7, Chapter 2, Definition 4, p. 23], there exists a least upper bound for $F$ which is contained in $B(m)$. Denote this least upper bound by $v$.

By the Radon-Nikodým theorem the map which sends $a$ in $D$ into $m_{a, x}$ in $M_{b}(V)$ may be regarded as a map sending $D$ into $L^{1}(v)$, carrying the unit ball of $D$ into a bounded set of $L^{\infty}(v)$. We denote this map by $T$. Further, if 
$h$ is the Radon-Nikodým derivative of $v$ with respect to $m$, then $L^{1}(v)$ may be imbedded in $L^{1}(m)$ by sending $f$ in $L^{1}(v)$ into $h f$. The composition of these two transformations is $\mathbb{E}$, carrying $D$ into $L^{1}(m)$.

Since $D$ is a refining space, $D^{\prime}$ is separable so that $D$ must be separable. By the Dunford-Pettis theorem [8], there exists a function $H$ mapping $V$ into $D^{\prime}$ such that:

(a) If $a$ is in $D$, then $T(A)=\langle a, H\rangle$.

(b) There exists a constant $k$ such that $\left|H_{\lambda}\right|_{D^{\prime}} \leqq k$ for almost all $\lambda$ in $V$. Or, defining $K=h \cdot H$, we have:

$\left(\mathrm{a}^{\prime}\right)$ If $a$ is in $D$, then $\subseteq(a)=\langle a, K\rangle$.

$\left(\mathrm{b}^{\prime}\right)\left|K_{\lambda}\right| D^{\prime} \leqq k h(\lambda)$ for almost all $\lambda$ in $V$.

Property $\left(\mathrm{b}^{\prime}\right)$ implies that the function $\lambda \rightarrow\left|K_{\lambda}\right|_{D^{\prime}}$ is integrable; that is, (5) is articulate over $D$.

\section{Generalized operator decomposition theorems in $L\left(D, D^{\prime}\right)$}

1. The decomposability of an algebra of operators over a refining Hilbert space. A positive measure $v$ over $V$, the spectrum of $A$, is called a basic measure for $A[7$, p. 112] if for any Borel set $B$ of $V$, then $v(B)=0$ if and only if $m_{x, x}(B)=0$ for all $x$ in $H$. This last is obviously equivalent to $E_{B}=0$. A measure $v$ with the property that $v(B)=0$ if and only if $m_{x, x}(B)=0$ for $x$ in a dense subset of $H$ is also a basic measure, since this last requirement is obviously equivalent to $E_{B}=0$. It is well known-a consequence of the separability of $H$-that there exists a bounded basic measure for $A$.

Let $D$ denote a refining space for $H$ which we now require to be a Hilbert space.

We may regard any continuous linear map in $L(H)$ as an element of $L\left(D, D^{\prime}\right)$ by first restricting the map to $D$ and identifying the elements of its range in $H^{\prime}$ with the elements of $D^{\prime}$ by the map $i^{*}$ taking $H^{\prime}$ into $D^{\prime}$. Through the above identification of the elements in $L(H)$ with those of $L\left(D, D^{\prime}\right)$, we may think of $G$ as a continuous linear map of $C_{0}(V)$ into $L\left(D, D^{\prime}\right)$. In the present part, $G$ will have only this meaning. Hence, $\left|T_{f}\right|$ denotes the norm of $T_{f}$ as a map from $D$ to $D^{\prime}$.

In what follows, we use the Schatten Theorem [20, Exposé no. 13, p. 5] which states that the space $L(D, D)$ is canonically isometrically isomorphic to the dual of $D \otimes{ }^{\wedge} D$. If we identify $D$ with $D^{\prime}$ by the natural identification of a Hilbert space with its antidual, then the Schatten Theorem may be read as stating that $L\left(D, D^{\prime}\right)$ is canonically isometrically isomorphic to the dual of $D \otimes \wedge D$. In what follows, we always assume that $L\left(D, D^{\prime}\right)$ is paired with $D \otimes{ }^{\wedge} D$, so that the $w^{*}$-topology over $L\left(D, D^{\prime}\right)$ is that which is induced by the pairing with $D \otimes \wedge D$.

Definition 3. A uniformly closed *algebra $A$ of operators acting on $H$ will be called decomposable over a refining space $D$ if there exists a bounded basic measure $v$ over the spectrum $V$ of the algebra and a $w^{*}$-measurable function $\phi$ mapping $V$ into $L\left(D, D^{\prime}\right)$ with the following properties: 
(a) The scalar function $|\phi|$ is essentially bounded with respect to $v$.

(b) The map $G$ carrying $C_{0}(V)$ into $L\left(D, D^{\prime}\right)$ is represented at any $f$ in $C_{0}(V)$ by the weak* integral $\int_{V} f \phi d v$.

We now state a theorem which is analogous in its purpose to Theorem 2, namely, it gives explicit criteria for an algebra to be decomposable over a refining space $D$.

Theorem 3. Let $A$ be a uniformly closed abelian *algebra and let $D$ be a refining space for $H$. Then the following assertions are equivalent:

(a) The family of measures $\left\{m_{a, a} \mid a\right.$ in $\left.S_{D}\right\}$ is bounded in the lattice structure of the space of bounded real Radon measures over $V$.

(b) There exists a bounded basic measure $v$ such that for each $f$ in $C_{0}(V)$, $|G(|f|)| \leqq v(|f|)$.

(c) The algebra $A$ is decomposable over $D$.

(d) The vector-valued set function sending a Borel set $B$ of $V$ into $E_{B}, E_{B}$ regarded as an element in $L\left(D, D^{\prime}\right)$, has the following two properties:

$1^{\circ}$ (Bounded variation). There exists a constant $c$ such that if $\left\{B_{i}\right\}$ is an arbitrary finite family of disjoint Borel sets of $V$, then

$$
\sum_{i}\left|E_{B_{i}}\right| \leqq c
$$

$2^{\circ}$ (Regularity). Let $C$ be an arbitrary Borel set of $V$ and $\epsilon$ an arbitrary positive number. There exists an open set $U$ containing $C$ such that for any Borel set $B$ in $U$,

$$
\left|E_{B}\right| \leqq \sup \left|E_{A}\right|+\epsilon
$$

where the supremum is taken over all Borel sets $A$ contained in $C$.

Proof. Assume (a); let $v$ be the least upper bound of $\left\{m_{a, a} \mid a\right.$ in $\left.S_{D}\right\}$. It is easy to see that $v$ is a basic measure for $A$. If $f$ is real and non-negative, then $|G(f)|=\sup _{a \in S_{D}}\langle a, G(f) a\rangle=\sup _{a \in S_{D}} m_{a, a}(f) \leqq v(f)$.

We now prove that (b) implies (c). Condition (b) states that $G$ is continuous if $C_{0}(V)$ is regarded as a subspace of $L^{1}(v)$ with the induced topology from $L^{1}(v)$. Therefore, there exists a continuous linear extension $J$ of $G$ mapping $L^{1}(v)$ into $L\left(D, D^{\prime}\right)$. On taking the transpose $J^{*}$ of $J$, we have that $J^{*}$ is a strongly continuous linear map from $D \otimes^{\wedge} D$ into $L^{\infty}(v)$. By the hypothesis that $D$ is a refining space, $D$ is separable, so that $D \otimes^{\wedge} D$ is separable. Applying the Dunford-Pettis Theorem [9], there exists a $w^{*}$-measurable map $\phi$ from $V$ to $L\left(D, D^{\prime}\right)$ with the following properties:

(a) The scalar function $|\phi|$ is essentially bounded with respect to $v$.

(b) If $x$ is an element of $D \otimes \wedge D$, then $J^{*}(x)=\langle x, \phi\rangle$. For any $f$ in $L^{1}(v)$ we have

$$
\langle x, J(f)\rangle=\left\langle J^{*}(x), f\right\rangle=\int_{V} f J^{*}(x) d v=\int_{V} f\langle x, \phi\rangle d v=\left\langle x, \int_{V} f \phi d v\right\rangle .
$$


So for any $f$ in $C_{0}(V), G(f)=\int_{V} f \phi d v$, where the integral is the weak* integral.

Assuming (c), $1^{\circ}$ of (d) follows from the fact that for an arbitrary finite family of disjoint Borel sets $\left\{B_{i}\right\}$,

$$
\sum_{i}\left|E_{B_{i}}\right| \leqq \int_{V}|\phi| d v
$$

so that we may set $c=\int_{V}|\phi| d v$. To prove $2^{\circ}$ we first note that for a given $\epsilon>0$ and a given Borel set $C$ of $V$, there exists an open set $U$ containing $C$ such that $\int_{U-C}|\phi| d v<\epsilon$. Hence, for any Borel set $W$ contained in $U-C$,

$$
\left|\int_{W} \phi d v\right| \leqq \int_{W}|\phi| d v \leqq \int_{U-C}|\phi| d v<\epsilon
$$

But any Borel set $B$ contained in $U$ is the union of a Borel set $W$ contained in $U-C$ and a Borel set $A$ contained in $C$; therefore,

$$
\left|\int_{B} \phi d v\right|=\left|\int_{W U A} \phi d v\right| \leqq\left|\int_{W} \phi d v\right|+\left|\int_{A} \phi d v\right| \leqq \epsilon+\left|\int_{A} \phi d v\right| .
$$

So, for any Borel set $A$ of $V$,

which proves $2^{\circ}$.

$$
\left|E_{B}\right| \leqq \epsilon+\sup _{A \subseteq C}\left|E_{A}\right|
$$

Assume (d). For any Borel set $B$ of $V$, we define $X(B)$ to be the supremum of the numbers $\left|E_{A}\right|$ for $A$ an arbitrary Borel set of $V$ contained in $B$. This determines a set function $X$ defined on the Borel sets of $V$ to the reals. In order to prove (a), it will be sufficient to show that $X$ satisfies conditions (a) through (e) of Theorem 1 where $F$ is the family of measures $\left\{m_{a, a} \mid a\right.$ in $\left.S_{D}\right\}$.

Positivity, sub-additivity, and monotonicity are clear. Let $\left\{B_{i}\right\}$ denote a finite family of disjoint Borel sets of $V$. If $A_{i}$ is an arbitrary Borel set of $V$ contained in $B_{i}$, then

$$
\sum_{i} X\left(B_{i}\right)=\sum_{i} \sup _{A_{i}}\left|E_{A_{i}}\right|=\sup _{A_{i}} \sum_{i}\left|E_{A_{i}}\right| \leqq c
$$

which proves that $X$ is of bounded variation. Regularity of $X$ follows from $2^{\circ}$ since condition $2^{\circ}$ states that for an arbitrary Borel set $C$ of $V$ and $\epsilon$ an arbitrary positive number, there exists an open set $U$ such that

$$
\sup _{B \subseteq U}\left|E_{B}\right| \leqq \sup _{A \subseteq C}\left|E_{A}\right|+\epsilon, \quad A \text { and } B \text { Borel sets of } V,
$$

that is, $X(U) \leqq X(C)+\epsilon$. Finally, for any Borel set $B$ of $V$ and any element $a$ in the unit ball $S_{D}$ of $D$,

$$
X(B) \geqq \sup _{A \subseteq B}\left|E_{A}\right| \geqq \sup _{A \subseteq B}\left\langle a, E_{A} a\right\rangle=m_{a, a}(B) .
$$


Hence, by Theorem 1, $\left\{m_{a, a} \mid a\right.$ in $\left.S_{D}\right\}$ is bounded in the lattice structure of the space of bounded real measures over $V$. This completes the proof of the theorem.

The following theorem is a convenient condition on the refining space for the decomposability of an algebra $A$ acting on $H$.

TheOREM 4. Let $D$ be a Hilbert space dense in $H$ as a vector space such that the natural map of $D$ into $H$ is nuclear. Then, $D$ is a refining space for $H$ and every uniformly closed abelian *algebra of operators acting on $H$ is decomposable over $D$.

Proof. We verify condition (a) of Theorem 3. Let $v$ be a bounded basic measure over the spectrum of the given algebra. We form the band $B(v)$ generated by $v$ in the space of bounded real Radon measures over the spectrum and recall that the Radon-Nikodým Theorem [6, Chapter 5, p. 47, Theorem 2] states that $B(v)$ is isomorphic to $L^{1}(v)$ in the topological and lattice structures of these spaces.

It is easy to see that the map sending $(x, y)$ in $H \times H$ into $\operatorname{Re} m_{x, y}$ is a real bilinear map of $H \times H$ into the space of real bounded Radon measures over $V$-which we denote by $M_{b}(V)$. Algebraically, this map may be identified with a real linear map of $H \otimes H$ into $M_{b}(V)$. For any $x$ and $y$ in $H$ the element $x \otimes y$ maps into Re $m_{x, y}$. Denote this algebraic map by $Y$. Now $Y$ is continuous if $H \otimes H$ is given the topology induced by the nuclear norm ||$_{1}$ (cf. Definition 1 of III). This follows from the fact that

$$
\left|\operatorname{Re} m_{x, y}\right| \leqq\left|m_{x, v}\right| \leqq\left|m_{x, y}(1)\right|=|\langle x, y\rangle| \leqq|x||y|=|x \otimes y|_{1}
$$

and so the unit ball of $H \otimes{ }^{\wedge} H$ is mapped into the unit ball of $M_{b}(V)$. Hence, there exists a continuous linear extension of $Y$, which we also denote by $Y$, mapping $H \otimes{ }^{\wedge} H$ into $M_{b}(V)$. Since $v$ is a basic measure, each measure $\operatorname{Re} m_{x, y}$ is a $B(v)$, so that the image of $Y$ in $M_{b}(V)$ may be identified with a subspace of $L^{1}(v)$.

Let $i$ denote the map of $D$ into $H$. Tensoring $i$ with itself, we have the transformation $i \otimes i$ carrying $D \otimes^{\wedge} D$ into $H \otimes \wedge H$. The linear operator $i \otimes i$ is nuclear [14, Chapter 2, p. 51 , Lemma 6]. Regarding $D \otimes^{\wedge} D$ as a subspace of $H \otimes \wedge H$, we restrict $Y$ to $D \otimes^{\wedge} D$; this restriction is nuclear, being the composition of a continuous operator and a nuclear operator. Hence, $Y$ maps the unit ball of $D \otimes{ }^{\wedge} D$ into a set bounded above in the lattice sense of $L^{1}(v)$ [14, Chapter 1, p. 64, Proposition 9]. But if $a$ is in the unit ball of $D$, then the element $a \otimes a$ is in the unit ball of $D \otimes{ }^{\wedge} D$, so that, using the identification of $L^{1}(v)$ with $B(v)$, we conclude that $\left\{m_{a, a} \mid a\right.$ in $\left.S_{D}\right\}$ is bounded in the lattice structure of $M_{b}(V)$.

The following proposition is a condition for the nuclearity of a map between two Hilbert spaces. 
Proposition 4. Let $E$ and $F$ be Hilbert spaces. Let $u$ be a mapping of $E$ into $F$ with the property that there exists an orthonormal basis $\left\{e_{i}\right\}$ in $E$ such that $\sum_{i}\left|u\left(e_{i}\right)\right|_{F}$ is finite. Then, $u$ is nuclear.

Proof. We see that the sum $\sum_{i} e_{i} \otimes u\left(e_{i}\right)$ converges absolutely in the nuclear norm, since

$$
\sum_{i}\left|e_{i} \otimes u\left(e_{i}\right)\right|_{1}=\sum_{i}\left|e_{i}\right|_{E}\left|u\left(e_{i}\right)\right|_{F}=\sum_{i}\left|u\left(e_{i}\right)\right|_{F}
$$

and the last sum is finite by hypothesis. Therefore, $\sum_{i} e_{i} \otimes u\left(e_{i}\right)$ represents a nuclear map of $E$ into $F$. However, for any $x$ in $E, \sum_{i}\left\langle x, e_{i}\right\rangle_{E} e_{i}$ converges in $E$ to $x$ since $\left\{e_{i}\right\}$ is a basis. So, $u(x)=\sum_{i}\left\langle x, e_{i}\right\rangle_{E} u\left(e_{i}\right)$ by continuity. On comparison, we have $u=\sum_{i} e_{i} \otimes u\left(e_{i}\right)$ which proves that $u$ is nuclear.

Proposition 4 yields a generalization of a theorem of Browder [5, Theorem 2] since Theorem 1 [5] is precisely the hypothesis of Proposition 4 above. That is, any abelian uniformly closed *algebra acting on $L^{2}\left(R^{n}, w\right), R^{n}$ Euclidean $n$-space and $w$ the Lebesgue measure, is decomposable over the particular Hilbert spaces that Browder constructs; the image of $\phi_{\lambda}$ may be represented by distributions of order at most $n+1$.

2. A decomposition theorem associated with a nuclear refining space. To my knowledge, Gelfand and Silov [13, footnote, p. 403] were the first to observe the importance of "nuclearity" in obtaining generalized eigenfunction expansions. The present section extends their theorem $[13$, p. 402 , Theorem $1]$.

Let $E$ be a linear topological space and let $U$ denote a convex and circled set of $E$. The space generated by $U$ in $E$ with the topology determined by the semi-norm derived from $U$ will be denoted by $E_{U}$. The completion of $E_{U}$ will be denoted by $E_{U}$. We now give a definition of a nuclear space which seems most convenient for us (cf. [20, Exposé 17, Theorem 1]).

Definition 4. A linear topological space will be called nuclear if for any convex circled and open neighborhood $U$ of zero in $D$ there exists another convex circled and open neighborhood $V$ of zero contained in $U$ such that the map taking $D_{V}$ into $D_{U}$ is nuclear.

We recall the following fact concerning nuclear spaces [14, Chapter 2, p. 37, Lemma 3]:

Lemma. A nuclear space $D$ possesses a fundamental family $\theta$ of open convex circled neighborhoods of zero such that for any $V$ in $\theta, D_{V}$ is a Hilbert space.

It is known that the injective map of a refining space $D$ of $H$ need not have an injective extension to the completion $\hat{D}$ of $D$. However, we may form the quotient of $\hat{D}$ with the kernel of the map of $\hat{D}$ into $H$ and then identify the elements of this quotient space with those of $H$; this resulting subspace of $H$ with the topology induced by the transfer to $H$ will be denoted by $[D]$. 
We remark that if $D$ is a normed space, the fact that the map of $D$ into $H$ is nuclear implies that the induced map of $[D]$ into $H$ is nuclear. This follows immediately from the representation of the map of $D$ into $H$ by $\sum_{i} \lambda_{i} x_{i} \otimes y_{i}$, the sequences $\left\{x_{i}\right\}$ and $\left\{y_{i}\right\}$ bounded in $D^{\prime}$ and $H$ respectively, and $\sum_{i}\left|\lambda_{i}\right|$ $<\infty[14$, Chapter 1, p. 51, Theorem 1] (cf. also [20, Exposé 12, p. 6, Propositions 7 and 8]).

THEOREM 5. Let $D$ be a nuclear refining space for $H$ with the additional property that for every open circled convex neighborhood $V$ of zero, the space $D_{V}$ is dense in $H$. Then, there exists an open convex circled neighborhood $W$ of zero such that $\left[D_{W}\right]$ is a refining Hilbert space for $H$ and every uniformly closed abelian *algebra of operators acting on $H$ is decomposable over $\left[D_{W}\right]$.

Proof. By Theorem 4 and the above remarks, it is sufficient to show that there exists an open convex circled neighborhood $W$ of zero such that the map of $D_{W}$ into $H$ is nuclear.

Since $D$ is a refining space, the map of $D$ into $H$ is continuous; hence, there exists, by Definition 4 , an open convex circled neighborhood $V$ of zero such that the map of $D_{V}$ into $H$ is nuclear. By the lemma, there exists an open convex circled neighborhood $W$ of zero contained in $V$ such that $D_{W}$ is a preHilbert space. Since the space $\left[D_{W}\right]$ is a Hilbert space-being the quotient of a Hilbert space by a closed subspace-it follows that Theorem 4 is applicable. This completes the proof of the theorem.

\section{BIBLIOGRAPHY}

1. W. G. Bade and J. T. Schwartz, On Mautner's eigenfunction expansions, Proc. Nat. Acad. Sci. U.S.A. vol. 42 (1956) pp. 519-525.

2. G. Birkhoff, Integration of functions with values in a Banach space, Trans. Amer. Math. Soc. vol. 38 (1935) pp. 357-358.

3. F. E. Browder, Eigenfunction expansions for singular elliptic operations. I, II, Proc. Nat. Acad. Sci. U.S.A. vol. 40 (1954) pp. 454-463.

4. - Eigenfunction expansions for formally self-adjoint partial differential operators. I, II, Proc. Nat. Acad. Sci. U.S.A. vol. 42 (1956) pp. 769-771, 870-872.

5. - Eigenfunction expansions for non-symmetric partial differential operators. I, Amer. J. Math. vol. 80 (1958) pp. 365-381.

6. N. Bourbaki, Intégration, Actualités Sci. Ind. nos. 1175 and 1244, Paris, 1952 and 1956.

7. J. Dixmier, Les algèbres d'opérateurs dans l'espace hilbertien, Paris, Gauthier-Villars, 1957.

8. N. Dunford and J. Pettis, Linear operations on summable functions, Trans. Amer. Math. Soc. vol. 47 (1940) pp. 325-392.

9. L. Gårding, Eigenfunction expansions connected with elliptic differential operators, Proceedings of the 12th Congress of Scandinavian Mathematicians, Lund, 1953.

10. - Applications of the theory of direct integrals of Hilbert spaces to some integral and differential operators, Lecture no. 11, Institute for Fluid Dynamics and Applied Mathematics, University of Maryland, 1954.

11. I. M. Gelfand, Abstrakte Functionen und lineare Operatoren, Mat. Sb. vol. 4 (1938) pp. 235-284. 
12. I. M. Gelfand and A. G. Kostyucenko, On expansions in characteristic functions of differential operators and other operators, Dokl. Akad. Nauk SSSR vol. 103 (1955) pp. 349-352.

13. I. M. Gelfand and G. E. Silov, Quelques applications de la theorie des fonctions genéralisées, J. Math. Pures Appl. vol. 35 (1956) pp. 383-413.

14. A. Grothendieck, Produits tensoriels topologiques et espaces nucleaires, Memoirs Amer. Math. Soc. no. 16, 1955.

15. P. R. Halmos, Measure theory, New York, Van Nostrand, 1950.

16. L. H. Loomis, $A n$ introduction to abstract harmonic analysis, New York, Van Nostrand, 1953.

17. F. I. Mautner, On eigenfunction expansions, Proc. Nat. Acad. Sci. U.S.A. vol. 39 (1953) pp. 49-53.

18. E. Nelson, Kernel functions and eigenfunction expansions, Duke Math. J. vol. 25 (1958) pp. 15-28.

19. J. von Neumann, On rings of operators, reduction theory, Ann. of Math. vol. 50 (1949) pp. $401-485$.

20. L. Schwartz, Séminaire Schwartz de la Faculté des Sciences de Paris, 1953-1954, Paris, Secrétariat Mathématique, 1954.

UNIVERSITY OF ILLINOIS,

URBANA, ILLINOIS

DUKE UNIVERSITY,

Durham, North Carolina 